EGU21-16385

https://doi.org/10.5194/egusphere-egu21-16385

EGU General Assembly 2021

(c) Author(s) 2021. This work is distributed under

the Creative Commons Attribution 4.0 License.

\title{
Cascading earthquakes on a fracture network in a geo-energy reservoir
}

\author{
Kadek Hendrawan Palgunadi ${ }^{1}$, Alice-Agnes Gabriel ${ }^{2}$, Dimitry Garagash ${ }^{3}$, and Paul Martin Mai ${ }^{1}$ \\ ${ }^{1}$ Physical Science and Engineering, King Abdullah University of Science and Technology, Thuwal, Saudi Arabia \\ (kadek.palgunadi@kaust.edu.sa,martin.mai@kaust.edu.sa) \\ ${ }^{2}$ Department of Earth and Environmental Sciences, Geophysics, Ludwig-Maximilians-Universität München, Theresienstr. 41, \\ 80333 Munich, Germany (gabriel@geophysik.uni-muenchen.de) \\ ${ }^{3}$ Department of Civil and Resource Engineering, Dalhousie University, Halifax, Nova Scotia, Canada (garagash@dal.ca)
}

The increasing rate of induced seismicity in subsurface reservoirs, exceeding occasionally moment magnitude 5, has generated significant attention among earthquake scientists and regulators over the last decade. Fluid injection activity during the operation stage often produces a significant, sometimes even destructive, earthquake. Many approaches have been proposed to monitor, model, and predict the injection-related seismicity to avoid an earthquake larger than a threshold set by the regulator (e.g., Mw 2.0). However, unexpected higher magnitude events occur exceeding what is predicted by empirical models, theoretical relations, or computer simulations.

Current models do not consider that subsurface reservoirs consist of complex fracture networks characterized by connected and unconnected individual fracture planes, often comprising a larger but inactive fault (unfavorably oriented with respect to regional stress). Fluid injection may then perturb stress conditions and trigger an initial rupture on fractures close to the injection well; this initial event may then dynamically trigger other fractures and potentially generate a large earthquake.

We inspect conditions leading to induced earthquakes taking into account the complex fracture network intersected to an inactive fault using dynamic earthquake rupture simulations. We generate the fracture network using a nearest-neighbor method following statistical parameters (power-law distribution of fracture length and fracture density) based on field data. There are 134 fractures consisting of 95 connected fractures, 3 fractures connected with at least one fracture, and 38 unconnected fractures. We focus on two fracture populations oriented in strike N110E \pm $10^{\circ}$ and $\mathrm{N} 210 \mathrm{E} \pm 10^{\circ}$, respectively. The main fault has a depth-dependent dip orientation which results in a listric fault geometry.

For our dynamic rupture simulations, we use the open-source software SeisSol (https://github.com/SeisSol/SeisSol), apply a laboratory-based rate-and-state with rapid velocity weakening friction law, and assign source radius-dependent characteristic length ( $L$ parameter) to the fractures. We vary stress conditions (maximum horizontal orientation, static-pore pressure, and prestress ratio) and conduct an initial static Mohr-Coulomb analysis before running the 
expensive dynamic rupture simulation. We choose conditions that lead to cascading rupture with (case 1) and without (case 2) the involvement of the main fault. Case 1 has higher artificial overstress within the nucleation area than case 2. Our simulation shows intricate rupture progression over small fractures via rupture branching with the parallel and orthogonal connected fractures. The rupture can also transfer to the unconnected fractures through dynamic triggering from the closest neighboring fracture. Case 1 produces a moment magnitude of Mw 6.36 that is equivalent to case 2 . Our preliminary result reveals that connected fractures can generate a significant and potentially large induced earthquake if all fractures are favorable to the stress condition. 